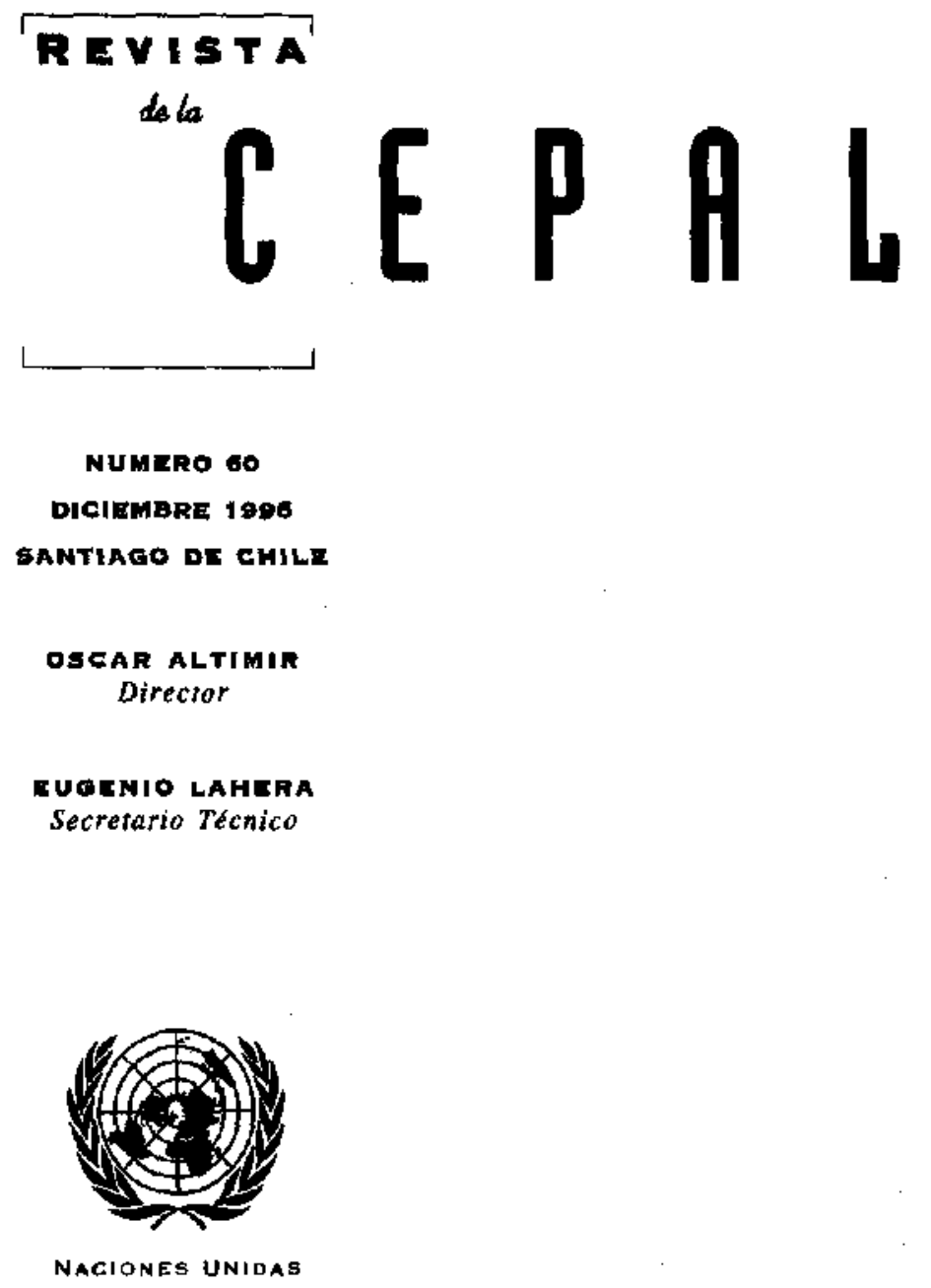


La evolución de las ideas y las políticas para el desarrollo

Gert Rosenthal

Políticas macroeconómicas para el crecimiento

Ricardo Ffrench-Davis

Flujos de capitales: lecciones a partir de la experiencia chilena

Eduardo Aninat, Christian Larrain

La transformación del desarrollo industrial de América Latina

José Miguel Benavente, Gustavo Crespi, Jorge Katz, Giovanni Stumpo

Las reformas de las pensiones en América Latina y la posición de los organismos internacionales

Carmelo Mesa-Lago

Aportes de la antropología aplicada al desarrollo campesino

John Durston

Prospección de la biodiversidad: potencialidades para los países en desarrollo

Julie M. Feinsilver

La inversión extranjera y el desarrollo competitivo en América Latina y el Caribe

Alejandro C. Vera-Vassallo

La crisis del peso mexicano

Stephany Griffith-Jones

Publicaciones recientes de la CEPAL 


\section{La evolución de las ideas y las políticas para el desarrollo}

\section{Gert Rosenthal}

Secretario Ejecutivo de la CEPAL
Este artículo entrega una visión de largo plazo del desarrollo de las ideas y políticas en América Latina y el Caribe. Examina los cambios de las tendencias en la formulación de políticas, señalando que los más abraptos de ellos ocurrieron, el primero en el períado de entreguerras, y el segundo a principios de los affos ochenta, y que en ambos casos se dieron cambios que pueden ser considerados pendulares o de etapas, pero que, en todo caso, fueron significativos. Enseguida pasa revista a las reformas economicas puestas en práctica a partir de los años ochenta, y pone de relieve sus factores comunes, los que sin embargo no corresponden a on paradigma, por las marcadas diferencias en la forma en que ellas se han aplicado en los diferentes países. Luego señala algunas enseñanzas extraídas del desempeño económico y social, entre las que destacan el insuficiente nivel de equidad logrado y el hecho de que los precios "correctos" son indispensables, pero insuficientes para el desarrollo. Pone de relieve la combinación de ortodoxia y heterodoxia que caracterizó a la experiencia de desarrollo de Asia oriental, y por último detalla diversas implicaciones de las tendencias actuales para el futuro regional, sobre todo la de asociar una gestión macroeconómica estable y coherente con una nueva forma de intervención a nivel microeconómico y mesoeconómico. 


\section{I}

\section{Introducción}

Analizar la evolución de las ideas y las políticas relacionadas con el desarrollo en América Latina y el Caribe; los efectos que han tenido esas políticas en los últimos años; las enseñanzas que se pueden extraer de ellos, y - como si todo lo anterior fuera poco- qué predicciones se podrían hacer sobre la situación futura en este campo, evidentemente no es una tarea fácil.

En primer lugar, el análisis de las estrategias de desarrollo en América Latina suele adolecer de un cierto maniqueísmo. El debate sobre el tema se reduce a alternativas aparentemente incompatibles; se habla de crecimiento "hacia adentro" o estrategias aperturistas, de laissez-faire o dirigismo, de estructuralismo o monetatismo. Pero la realidad es mucho más compleja y la variedad de enfoques aplicados cubre un espectro de políticas y estrategias bastante amplio; por lo tanto, no podemos limitarnos a un solo paradigma.

Lo mismo se podría decir de las relaciones de causa y efecto. A veces, se considera que el desempeño económico de un país sólo depende de factores determinados por la situación económica internacional; en otros casos, en cambio, el análisis se limita únicamente a los efectos de las políticas internas. También en este caso se podría decir que la realidad es mucho más compleja, y que las distintas formas que adopta la formulación de las políticas y las relaciones de causalidad no pueden describirse en términos categóricos, en blanco y negro, porque de hecho corresponden a distintos matices de gris.

En segundo lugar, una vez más hay que tener presente que América Latina está integrada por países muy distintos. Ni las modalidades de los affos cincuenta ni las de los años ochenta se aplicaron por igual en todos ellos; algunos países adoptaron un determinado modelo antes que otros $\rightarrow 0$ con más entusiasmo-, y se puede observar grandes diferencias en la formulación de las políticas en lo que respecta a su ritmo, a la sucesión cronologica y a la combinación de medidas. Además, la aplicación de políticas similares en distintos países ha dado, naturalmente, resultados dispares; no podía haber sido de otra manera, puesto que cada contexto es diferente. Por lo tanto, siempre es arriesgado intentar sacar conclusiones de validez universal de la enorme variedad de casos que la region ofrece.

A pesar de estas reservas, he hecho un esfuerzo por atenerme a los temas señalados, con la idea de destacar ciertos aspectos y de referirme a parámetros que pueden facilitar el debate sobre las diferencias que se han ido dando, con el correr de los años, en la formulación de las políticas económicas y sociales en América Latina, y de comparar esa evolución con la de otras regiones en desarrollo. En las secciones siguientes, necesariamente breves, examino los cambios en la formulación de políticas (sección II); paso revista a las reformas económicas realizadas a partir de la década de 1980 (sección III); intento sacar algunas conclusiones de la situación que se ha dado en el ámbito económico y social, en la medida en que ésta se vincula a las reformas (sección IV); comparo brevemente lo sucedido en Asia oriental y America Latina (sección V), y por último, hago un sondeo del futuro (sección VI).

\section{II}

\section{Tendencias y cambios en las políticas}

Se podría decir que el pensamiento económico está sujeto a un proceso comparable a los ciclos que carac-

Este articulo recoge en lo medular la intervención del autor en la Conferencia sobre el Pensamiento y la Práctica en materia de Desarrollo (Washington, D.C., 3 a 5 de septiembre de 1996), organizada por el Banco Interamericano de Desarrollo (BID). terizan a la evolución de la economía. El equivalente de las ondas de Kondratieff - los ciclos largos-es un reflejo de las tendencias teóricas predominantes en una determinada época, en lo que respecta a dos temas interrelacionados, el papel económico del gobierno (desde La riqueza de las naciones de Adam Smith 
hasta El fin del laissez-passer de Keynes) y el grado de "apertura" al comercio internacional. Los ciclos más cortos, entonces, serían un producto de los excesos que suelen cometerse cuando se aplican en la práctica las políticas que surgen del mundo de las ideas. En otras palabras, las acciones están determinadas por las ideas así como las ideas se ven influidas por las acciones.

América Latina ciertamente ha atravesado por estos ciclos en los últimos ochenta años, tanto en el terreno del pensamiento económico como en el de la gestión económica. Ha habido por lo menos dos giros abruptos: el primero entre el período de entreguerras y el período posbélico, como reacción a la Gran Depresión y a los efectos de la segunda guerra mundial; el segundo en el decenio de 1980 , como reacción a la crisis de la deuda y a la globalización. Hay autores que hablan de un tercer cambio, que se habría producido a mediados de los años setenta, al menos en el Cono Sur, pero trataré ese tema más adelante. En cada uno de esos casos ha habido también una constante evolución, tanto en el plano conceptual como en la práctica.

Este cambio ininterrumpido, que algunos prefieren considerar un movimiento pendular y otros describen como "etapas" (Birdsall y Lozada, 1996), refleja una combinación de tendencias universales y de reacciones propias de cada país a las crisis externas y los acontecimientos internos. Además, al examinar esta dinámica es difícil saber si el pensamiento económico y la aplicación de las políticas condujeron a una determinada situación en el ámbito económico o si el pensamiento económico estuvo más bien determinado por la realidad económica; de hecho, por supuesto, ambos fenómenos se dan en forma simultánea.

El primer giro importante al que me he referido se produjo en los años treinta y cuarenta, período en el que la mayoría de los países de la región abandonaron los planteamientos ortodoxos para adoptar una estrategia de industrialización basada en la sustitución de importaciones junto con las políticas intervencionistas que ésta suponía. Los factores económicos que impulsaron este cambio derivan de la crisis de los años treinta, puesto que los países comenzaron a hacer frente a la grave depresión y la restricción de divisas que ésta había provocado, restringiendo las importaciones y estimulando la oferta interna. Una vez superado el problema de escasez de divisas gracias a la acumulación de reservas durante la segunda guerra mundial, la dificultad para adquirir bienes manufacturados en los mercados internacionales impuso la necesidad de seguir aplicando el modelo de sustitución de importaciones, especialmente en los países más grandes.
Como se sabe, el marco conceptual de esta tendencia surgí, al menos en parte, en la CEPAL, encabezada en ese entonces por don Raúl Prebisch, quien desarrolló un argumento muy convincente en favor de la industrialización y de una presencia estatal fuerte, basado en las relaciones asimétricas entre "centro" y "periferia" (CEPAL, 1951). En dicha teoría se ponía el acento en los factores estructurales que dificultaban el desarrollo, es decir, prácticamente en la antítesis de la "magia del mercado". La reflexión tenía un alcance de mediano y largo plazo; en general, se otorgó poca importancia a la formulación de políticas económicas de corto plazo, especialmente en el terreno de las políticas monetaria, cambiaria y fiscal.

Aunque el mismo Prebisch advirtió contra el peligro que podría encerrar una protección excesiva, con el paso de los años se estereotipó el "mensaje" de la CEPAL, que ha sido descrito como excesivamente "orientado hacia adentro" y "dirigista", De hecho, en la región surgieron importantes grupos de presión -entre otros, cámaras de industria y sindicatos-, que eran partidarios de mantener la protección e incluso de acentuarla, lo que creaba una dinámica que se autoperpetuaba y que otros grupos de presión —cámaras de comercio y agricultura, por ejemplo— no lograban mitigar. Por lo tanto, se podría afirmar que la estrategia de sustitución de importaciones se siguí aplicando mucho tiempo más allá de lo que aconsejaba la prudencia. Por otra parte, el desempeño económico de la región en las décadas de 1950 y 1960 , al menos desde el punto de vista del crecimiento, fue bastante respetable: el promedio del PIB per cápita real prácticamente se duplicó entre 1950 y 1970.

No es sorprendente, entonces, que la oposición al enfoque aplicado por la CEPAL en los años cincuenta -proveniente de los círculos académicos de América Latina (algunos ejemplos notables son Viner, 1953, y Corden, 1966) y también de las instituciones de Bretton Woods- fuera discreta al comienzo, pero que luego cobrara mucha fuerza en la década de 1960 y despertara serias dudas en ambos extremos del espectro ideológico. Los "dependentistas" pedían implícitamente más intervención estatal y menos dependencia del comercio y las corrientes de capital; los más conservadores abogaban por una menor intervención del Estado y medidas económicas más ortodoxas. Además, la industrialización apoyada en la sustitución de importaciones se enfrentó a ciertos problemas en la práctica, debido a su sesgo antirrural y antiexportador y a la comprobación de que de hecho acentuaba la vulnerabilidad externa en vez de mitigarla. 
Al mismo tiempo, fuera de América Latina se iba produciendo un cambio en el pensamiento económi$\mathrm{co}$, que, como es lógico, influía en el debate regional. Aunque la discusión sobre "apertura" al comercio internacional no era el aspecto más importante del debate, los principios económicos poskeynesianos de la corriente dominante eran criticados tanto por los pensadores conservadores (la escuela de Chicago con sus postulados libertarios) como por pensadores progresistas (los "economistas radicales"). Sin embargo, el modelo siguió vigente en América Latina durante otros diez años, más o menos, gracias a varios fenómenos: i) la ampliación de los mercados nacionales con la adopción de acuerdos formales de integración económica; ii) la reducción del sesgo antiexportador mediante la aplicación de políticas de fomento de las exportaciones no tradicionales, sobre todo de la concesión de incentivos fiscales, y iii) el mayor acceso al financiamiento internacional, sobre todo el proveniente de fuentes públicas en los años sesenta y de fuentes privadas en los setenta.

Como se sabe, la contrarrevolución más radical se produjo a mediados de la década de 1970 , y al comienzo se limitó solamente a Argentina, Chile y Uruguay. Los países del Cono Sur sufrieron en esa época graves desequilibrios macroeconómicos y distorsiones de precios; los tres tenfan gobiernos militares autoritarios, y los tres volvieron a lo que un autor llama "una economia neoconservadora" (Ramos, 1986) y otro denomina "monetarismo internacional" (Fishlow, 1985). Como dice Fishlow, "el objetivo inicial era reducir la inflación interna, mediante ajustes predeterminados y decrecientes del tipo de cambio, aplicando la ley del precio ánico. Una vez reducida la inflación, se adoptaba un tipo de cambio fijo para evitar que volviera a subir. El libre comercio y los movimientos de capital facilitarían la estabilización y garantizarían el desarrollo" (p. 135).

Sin embargo, otros aspectos del enfoque aplicado en el Cono Sur son presagios de lo que sucedería en los años ochenta (Corbo, 1988): la liberalización del comercio y de los mercados financieros internos, la apertura a las corrientes internacionales de capital, la desregulación, la reducción del déficit fiscal (menos en Argentina que en los otros países) y la adopción de políticas monetarias restrictivas. En general, se restableció la libertad de precios como principal mecanismo para la asignación eficaz de los recursos, aunque como bien señala Albert Fishlow "el monetarismo internacional no funciono como se había previsto", debido a tres limitaciones importantes: "la aplicacion al corto plazo de condiciones de equilibrio de largo plazo; la escasa atención prestada a los componentes de la balanza de pagos, porque lo único que interesaba eran las cifras globales, y el énfasis en el equilibrio macroeconómico en desmedro del desarrollo económico" (pp. 136-137). En efecto, los tipos de cambio sobrevalorados y la notable afluencia de capitales de corto plazo, así como la poca importancia otorgada a la oferta en la ecuación oferta-demanda, se tradujeron en una profunda recesion y un sistema bancario insolvente.

Todo esto supuso una ruptura definitiva con la estrategia aplicada en el pasado por los países del Cono Sur; según Fishlow, la corrección de los defectos más evidentes del nuevo enfoque exigía también nuevos criterios en lugar de retomar los antiguos, aunque existía cierto conflicto entre los defensores de cada una de estas opciones. ${ }^{1}$ La crisis de la deuda de 1982 provocó un importante cambio en el pensamiento económico y la formulación de políticas, no sólo porque las circunstancias lo imponían, sino también debido a que la nueva y grave crisis de la balanza de pagos dejó en evidencia las deficiencias de la estrategia anterior (su vulnerabilidad a las crisis internacionales, las distorsiones de precios y la falta de competitividad internacional), que se habían ido acumulando gradualmente a lo largo de los años.

Al comienzo, el ajuste y la estabilización se abordaron en forma poco sistemática y con un costo social extremadamente alto. En la primera mitad de la década de 1980 se dio una curiosa mezcla de control y liberalización, de métodos ortodoxos y heterodoxos. Los programas de ajuste "heterodoxos" de Argentina, Brasil y Perú despertaron un enorme entusiasmo; su objetivo era controlar la inflación sin caer en una recesión, orientando las expectativas a través de los controles de precios e ingresos a corto plazo $\mathrm{y}$, a la vez, liberalizar los mercados a mediano plazo. A diferencia de lo ocurrido en Israel en el mismo período, todos estos programas fracasaron en último término, por distintos motivos (Bruno y otros, 1988), ${ }^{2}$ aunque paralelamente

\footnotetext{
' El conflicto se teflejo también en esa época en la Secretaría de la CEPAL, donde se dio un intenso debate entre los partidarios de ambas estrategias.

${ }^{2}$ Hay quienes también consideran a Bolivia como un ejemplo exitoso de estabijizaxión heterodoxa. Sin embargo, dado el nivel de inflación que registraba Bolivia en ese entonces (1985), la ortodoxia y la heterodoxia tienden a coincidir en materia de políticas (Morales y Sachs, 1988). El Plan Real de Brasil representa un intento más actual de aplicación de un programa heterodoxo, pero con mayor control fiscal que los descritos.
} 
se dio un proceso de aprendizaje y se produjo una convergencia gradual con respecto a los principales parámetros de un nuevo enfoque, que indudablemente representa un acercamiento a una mayor ortodoxia.

\section{III}

\section{Las reformas de los últimos años}

La convergencia mencionada se manifiesta en varios aspectos fundamentales de la formulación de políticas, entre otros una evidente tendencia a la liberalización, una mayor confianza en las fuerzas del mercado como principal mecanismo de asignación de recursos, la adopción de políticas públicas menos "intervencionistas" y una mayor coherencia de las políticas macroeconomicas. Así como la crisis de los años treinta dio origen al modelo de sustitución de importaciones y a una mayor intervención del sector público, la crisis de los años ochenta marca el comienzo de un desplazamiento hacia economías más abiertas y orientadas al mercado.

Algunos autores han interpretado este cambio en el pensamiento económico como el surgimiento de un nuevo paradigma, que los medios de comunicación latinoamericanos suelen definir como "neoliberal". En términos más sencillos, hace algunos años John Williamson se refirió a este marco de formulación de políticas como "el consenso de Washington" (aunque no fue concebido en Washington y no responde a un consenso; el mismo Williamson dice que lamenta haber empleado ese término). Este enfoque se ha reflejado en algunas reformas fundamentales de las políticas y los mercados, y tiene un doble objetivo de estabilización macroeconómica y desarrollo de la competitividad internacional.

En términos más concretos, y al margen de las diferencias de grado y prioridades entre un caso y otro, hay varios elementos de este enfoque que se repiten en todos los casos: i) adopción de medidas explícitas para liberalizar el comercio, caracterizadas por la eliminación de las restricciones cuantitativas y la fijación de aranceles moderados dentro de márgenes limitados $o$, de preferencia, un arancel uniforme bajo; ii) disciplina fiscal (reducción del déficit del sector público e incluso logro de un superávit), además de una reorientación y priorización de los gastos públicos; iii) una reforma tributaria (ampliación de la base tributaria, perfeccionamiento de los mecanismos administrativos, abolición de las exenciones especiales); iv) disciplina monetaria que incluye la adopción de tasas de interés real moderadas pero positivas; v) liberalización financiera basada en la abolición gradual del cobro de tasas de interés preferenciales a prestatarios privilegiados; vi) privatización de la mayoría de las empresas estatales y desregulación consistente en la eliminación de todas las disposiciones que restrinjan la competencia (salvo en el caso de monopolios naturales u otras situaciones excepcionales); vii) desregulación de los mercados financieros y laborales; viii) eliminación de las barreras impuestas al ingreso de inversiones extranjeras directas, y ix) marco jurídico e institucional que respalde la observancia de los derechos de propiedad (Williamson, 1990).

En los últimos años, algunos expertos han comenzado a hablar de una "segunda generación" de reformas, que incluiría lo siguiente: la creación de bancos centrales independientes; la implantación de restricciones presupuestarias a los gobiernos estatales, provinciales y locales; la creación de una administración pública nacional moderna y eficaz; el realce de la seguridad ciudadana, y una reforma del sistema judicial (Edwards, 1996).

Tan importante como lo anterior son los elementos no incluidos en el enfoque descrito, puesto que la desmesurada importancia otorgada a las señales del mercado como base para la asignación de recursos se tradujo en la virtual desaparición de los incentivos a determinadas áreas de actividad, de los planes de fomento de las inversiones y, sobre todo, de las medidas de política industrial. De hecho, hay quienes sostienen que el enfoque es una herramienta de estabilización, no una estrategia de desarrollo. En todo caso, las autoridades no sólo se concentraron en la formulación de políticas de corto plazo, sino que también aceptaron tácitamente la idea de que las empresas de algún modo se adaptarían al nuevo marco regulador y a los incentivos macroeconómicos, lo que a la larga les permitiría adquirir competitividad internacional por medio de la "magia del mercado".

Por otra parte, este enfoque difícilmente podría considerarse un paradigma. De hecho, hay profundas 
diferencias entre un país y otro en cuanto a la aplicación práctica de sus componentes; a su orientación concreta; al ritmo, el alcance y la secuencia de la implementación y a la combinación de políticas utilizadas ("menú de políticas").

Se pueden citar tres ejemplos muy ilustrativos. En primer lugar, lo que ha ocurrido en el ámbito de las políticas cambiarias. Si bien todos los países comparten los objetivos de unificar los tipos de cambio y darles estabilidad y competitividad, han adoptado métodos diferentes, que van desde la adopción de un tipo de cambio flotante relativamente libre (dentro de ciertos límites), pasando por una flotación "sucia" y una flotación preestablecida y controlada, hasta el establecimiento de un tipo de cambio fijo. ${ }^{3}$ En segundo lugar, las variadas características que presentan los programas antiinflacionarios aplicados en la región, que se diferencian sobre todo en lo que respecta al ritmo de implementación y a la complementación, o no complementación, de las restricciones monetarias y fiscales convencionales con políticas de precios e ingresos destinadas a influir en las expectativas de los agentes económicos. Estas diferencias explican la coexistencia en la región de programas de aplicación gradual, acompañados de indización de los precios, y de programas "de choque". El tercer ejemplo se encuentra en la liberalización de la cuenta de capital, proceso que en algunos países se lleva a cabo paralelamente a la liberalización de la cuenta corriente, mientras en otros éstos se realizan en forma secuencial (Ffrench-Davis, Titelman y Uthoff, 1994).

Hay muchas otras areas en las que se observan diferencias en la formulación de las políticas; entre otras, cabría mencionar las siguientes: i) medidas orientadas a contrarrestar los efectos regresivos de las politicas económicas (focalización del gasto público, transferencias); ii) medidas destinadas al fomento de determinadas actividades productivas; iii) voluntad política de privatizar las empresas públicas, especialmente las dedicadas a la explotación de recursos minerales de exportación (petróleo en México, cobre en Chile); iv) medios empleados para reglamentar los monopolios de los servicios públicos privatizados; v) modificación del marco jurídico e institucional de los derechos de propiedad; vi) adopción de normas jurídicas congruentes con la meta de disciplina fiscal y monetaria;

\footnotetext{
${ }^{3}$ Cabe señalar, sin embargo, que ningún país ha adoptado un tipo de cambio sin intervención del Banco Central, ni siquiera aquellos donde funcionan mercados a futuro de moneda nacional.
}

vii) inclusión en los procesos de reforma tributaria de las metas de equidad social y eficiencia en ta asignación de los recursos; viii) contenido y alcance de las reformas de la seguridad social, las prestaciones sociales y las leyes laborales, y ix) en general, contribución del sector público al producto interno bruto, que varía de $10 \%$ a $30 \%$.

Estos elementos, unidos a la diversa orientación política de los equipos gobernantes, las cambiantes características de los sistemas políticos y los diferentes grados de madurez económica y tecnologica de los sistemas productivos de los distintos países, explican no sólo las diferencias de ritmo y contenido de las reformas, sino también los diversos resultados obtenidos.

En resumen, las reformas institucionales más importantes realizadas por la mayoría de los países de la region han supuesto un profundo cambio en la formulación de políticas, y en el nivel y atcance de la intervención estatal en la economía. Este cambio aceleró la transformación de la modaljdad de desarrollo, que ya se vislumbraba antes de la crisis de la deuda, y determinó su curso. Es un cambio que implica la consideración de las exportaciones y la inversión privada como principales motores del crecimiento, función que antes se otorgaba a la sustitución de importaciones y las inversiones públicas. Sin embargo, la forma en que se ha instrumentado este enfoque general ha variado en gran medida de un país a otro.

El último aspecto de la formulación de políticas en la década de 1980 al que quisiera referirme son los acuerdos oficiales de integracion económica. Como se sabe, en los años cincuenta y sesenta se firmaron varios acuerdos de libre comercio o de creación de mercados comunes, que contribuyeron en gran medida a la industrialización con fines de sustitución de importaciones, y que fueron objeto de múltiples críticas, al igual que la estrategia. Durante la primera mitad de los años ochenta, estos acuerdos prácticamente desaparecieron del panorama regional, para reaparecer años más tarde bajo un aspecto diferente, el de una segunda generación de acuerdos, más compatibles con las estrategias orientadas a las exportaciones, que son los que se han adoptado en el último decenio. Esta forma de "regionalismo abierto" (CEPAL, 1994) ha cobrado ímpetu en los años noventa e incluso ha abierto una posibilidad de integración continental con la firma del Tratado de Libre Comercio de América del Norte entre Canadá, México y los Estados Unidos, y el posterior compromiso asumido por los Jefes de Estado y de Gobiemo en el sentido de crear un "área de libre comercio de las Américas" a partir del año 2005. 


\section{IV}

\section{Enseñanzas de las reformas recientes}

¿Qué problemas socioeconómicos importantes fueron superados gracias a las políticas aplicadas en la década de 1980 y qué enseñanzas nos dejan? A pesar de lo difícil que es establecer relaciones causales entre pensamiento y acción, entre las políticas y sus efectos, no cabe duda de que la década de 1990 presenta ciertas características destacadas y que es posible llegar a algunas conclusiones generales (CEPAL, 1996a y 1996b).

En cuanto a los efectos, la adopción de un enfoque más orientado al mercado y la mayor coherencia y estabilidad de la gestión macroeconomica, especialmente en las esferas fiscal y monetaria, obviamente han contribuido de manera importante al control de la inflación. Durante la segunda mitad de los años ochenta, varios países (Argentina, Bolivia, Brasil, Perú, Nicaragua) se vieron afectados por una hiperinflación; el promedio ponderado de la inflación anual de la región fue del orden del $1000 \%$ en 1988, 1989 y 1990 . En 1995 , ese promedio fue de $25 \%$, y cerca de la mitad de los países había reducido la inflación anual a alrededor del $10 \%$ o menos. Es innegable que esto obedecio a las políticas de estabilización, que influyeron tanto en las expectativas de los agentes económicos como en la demanda agregada.

Además, a comienzos de la los años noventa, la mayoría de los países de la región había recuperado la capacidad de crecer, aunque a tasas moderadas. Algunos crecieron rápidamente durante uno o dos años, pero luego se enfrentaron a la necesidad de un ajuste; Chile ha sido prácticamente el único que ha conseguido mantener altas tasas de crecimiento durante un largo período. El hecho de que el crecimiento no haya sido más dinámico refleja, en parte, las compensaciones recíprocas entre las políticas orientadas a mantener la estabilidad y las destinadas a fomentar el crecimiento, materia que sigue siendo objeto de una intensa polémica (Dornbusch y Werner, 1994).

Otra manifestación de posibles compensaciones recíprocas entre crecimiento y estabilidad es el fenómeno que se dio en el sector externo, cuando los países de la región dejaron de registrar un superávit en cuenta corriente para enfrentarse a un déficit cada vez mayor. En efecto, a partir de 1991 el crecimiento moderado con una creciente estabilidad de precios se vio reforzado por una notable afluencia de capital in- ternacional, atraído en parte por las altas tasas de interés y, en parte también, por la confianza que inspiraban el mejoramiento de la gestión macroeconómica, los adelantos logrados mediante reformas estructurales y la consolidación de regímenes políticos democráticos. Evidentemente, éste es un logro notable, si se compara con el prolongado período de transferencia neta de recursos financieros que había vivido la región pocos años antes.

La afluencia de capital en 1993 y 1994 fue de tales proporciones que, en muchos países, contribuyó a la apreciación del tipo de cambio y obligó a las autoridades monetarias a elevar aún más las tasas de interés. Este síndrome, combinado con políticas internas inadecuadas, fue una de las causas de la crisis financiera mexicana de diciembre de 1994 (Sachs, Tornell y Velasco, 1995) y recordó a los responsables de la formulación de políticas que el mantenimiento de los equilibrios macroeconómicos es un constante desafío. Lo anterior significa que las condiciones en las que se enmarca la evolución de una economía son un factor de vulnerabilidad. En muchos casos, la estabilidad macroeconómica se ha logrado a costa de un voluminoso déficit en cuenta corriente de la balanza de pagos, financiado en algunos casos con capital volátil, que puede retirarse al menor asomo de cambios que reduzcan la confianza de los inversionistas.

Lo que empeoro esta difícil situación fue el hecho de que el cuantioso ahorro externo no se encauzara a una mayor inversión; por el contrario, gran parte de éste se destinó a la importación de bienes de consumo. La relación entre ahorro nacional y producto interno bruto había ido aumentando lentamente, mientras la relación entre inversión total y PIB seguía siendo mucho menor que la registrada antes de la crisis de la deuda. En el caso de México, por ejemplo, el ahorro externo aumentó de un $3.6 \%$ a un $7.0 \%$ del PIB entre 1990-1991 y 1993-1994, mientras el ahorro nacional disminuía del $18.5 \%$ al $15.6 \%$ (CEPAL, 1996b, p. 53).

Otra manifestación de la vulnerabilidad que aún subsiste, a pesar de los progresos logrados, es la que se observa en dos dimensiones estructurales. La primera es la escasa solidez de los sistemas financieros de algunos países, que suele verse agravada por las deficiencias de los organismos fiscalizadores; las nu- 
merosas crisis financieras que afectan a grandes instituciones bancarias han obligado a realizar operaciones de rescate que han tenido un alto costo fiscal. La segunda dimensión es la exigua estabilidad fiscal. De hecho, los avances logrados han tenido cimientos muy frágiles; la contracción económica que sufrieron $\mathrm{Ar}$ gentina y México en 1995 estuvo acompañada de una baja desproporcionada de los ingresos fiscales, que forz 6 a los gobiernos de ambos países a elevar los impuestos.

Sin embargo, es posible que la limitación más evidente del desempeño económico de los últimos años, que sin duda está vinculada a las moderadas tasas de crecimiento, sea la que atẫe a la equidad. Aunque la incidencia de la pobreza ha disminuido gradualmente en la mayorfa de los paises, aún se mantiene a un nivel mucho más alto que el de 1980 , en tanto que la distribución del ingreso ha empeorado en casi todos los países (Uruguay parece ser la principal excepción), ${ }^{4}$ salvo en los que han registrado un rápido descenso de la inflación, como es el caso de Brasil en 1994-1995. Los escasos adelantos logrados en el plano de la equidad (CEPAL, 1995a) pueden atribuirse a tres fenómenos.

En primer lugar, la reestructuración del aparato productivo derivada del ajuste, las variaciones de los precios relativos y la liberalización del comercio no han logrado hasta la fecha generar suficientes empleos para absorber a los nuevos contingentes que se incorporan a la población económicamente activa. En la actual etapa de crecimiento moderado, las oportunidades de empleo productivo han ido aumentando muy lentamente, mientras las disparidades salariales entre empleos que exigen distintos grados de capacitación han tendido a ampliarse.

En segundo lugar, las políticas públicas adoptadas hasta ahora con el objeto de aliviar las manifestaciones más graves de pobreza y marginalización no han contado con los recursos necesarios para lograr sus objetivos y han adolecido de una limitada eficacia; el gasto social se ha restringido, a raiz de los intentos por imponer una disciplina presupuestaria y la gestion de las políticas sociales no ha progresado con la agilidad y eficacia deseadas.

Por últìmo, aunque el veredicto final está lejos de ser definitivo - hay un amplio debate al respecto

\footnotetext{
"Se podría conjeturar que Uruguay ha sido una excepción en este sentido, en parte debido a que no ha desmantelado el antiguo $y$ generoso sistema de previsión social, legado del Estado benefactor cuya existencia se opone abiertamente a las tendencias predominantes en las últimas décadas.
}

(Scobie, 1990; Taylor, 1988)—, hay indicios de que, salvo en los países que redujeron drásticamente la inflación, la formulación de las políticas ha tenido un sesgo regresivo, dados los efectos iniciales del ajuste en los salarios reales, las tasas reales de interés, el nivel de empleo y el gasto público.

El deterioro de la equidad ha quedado aún más en evidencia en el plano microeconómico, en el que los procesos de ajuste, de estabilización macroeconómica y de reforma estructural han actuado como un poderoso mecanismo de selección, que ha forzado a las empresas a adaptarse a las nuevas circunstancias, regidas por las señales del mercado y las nuevas modalidades de regulación de las actividades productivas. No resulta sorprendente, entonces, que diferentes empresas hayan reaccionado de distìnta manera a estos fenómenos, dependiendo de la actividad a la que se dedican, la region en la que se encuentran y sus sistemas de produccion. En general, los sectores más perjudicados por los cambios en las reglas del juego y el marco regulatorio han sido las industrias orientadas al mercado interno, las actividades que suponen un uso relativamente intensivo de servicios de ingeniería, las empresas pequeñas y medianas $y$, en general, las empresas estatales. En cambio, los sectores dedicados a actividades de exportación o basadas en recursos naturales, los grandes conglomerados nacionales y muchas empresas transnacionales han logrado adaptarse a las cambiantes circunstancias con mejores resultados,

La reestructuración ha obligado a los sistemas productivos de la región a regresar a las áreas que tradicionalmente han ofrecido ventajas comparativas, es decir los productos primarios e industriales, aunque en algunos casos han conseguido incorporar nuevas tecnologias que les han dado mayor acceso a los recursos naturales disponibles y adoptar métodos más modernos de explotación. La heterogeneidad estructural característica de los sistemas productivos de la región se ha acentuado, como consecuencia de la tendencia a la ampliación de las diferencias en términos de productividad entre las grandes empresas que se ubican en la frontera tecnológica a nivel mundial y las numerosas y variadas actividades que van quedando rezagadas. Esta tendencia se ha intensificado cada vez más a raíz de la segmentación del crédito, que prácticamente ha inpedido a las pequerias y medianas empresas accecer a las fuentes más importantes de capital. Otro reflejo de este fenómeno es la rápida desaparición de las tradicionales grandes tiendas, debido al surgimiento de centros comerciales similares a los de Estados Unidos, 
administrados por conglomerados nacionales o internacionales. En último término, esto ha significado que, mientras la productividad de los factores y de la mano de obra ha aumentado aceleradamente en los sectores "modernos", ésta ha disminuido en los sectores menos avanzados, lo que ha neutralizado en parte el progreso logrado por la economía en general.

En resumen, los cambios realizados en materia de formulación de políticas en la década de 1980 no se han visto complementados por un desempeño económico realmente satisfactorio, aunque no se puede dejar de reconocer que, en comparación con la desastrosa década anterior, se han logrado avances importantes. Además, teóricamente el proceso de reforma debería dejar a los países mucho más capacitados que antes para responder a los desafíos de la globalización. Sin embargo, los adelantos que ésta ha hecho posible no han tenido la solidez ni el alcance necesarios para lograr un crecimiento sostenido, una estabilidad financiera y un incremento de la equidad. En todo caso, la experiencia adquirida contribuye a la evolución de las ideas y la formulación de políticas en el plano económico.

Entre las lecciones más importantes de este proceso, destacan dos ideas articuladoras: En primer lugar, la estabilidad macroeconómica parecería ser una condición esencial para estimular el ahorro y la inversión, y para elevar la tasa de crecimiento. A fin de que la demanda efectiva corresponda a la frontera productiva y de amortiguar los efectos de las conmociones de origen externo, los países necesitan políticas monetarias, crediticias, físcales y comerciales que sean coherentes entre sí, aunque evidentemente diferirắn de uno a otro en cuanto a su alcance y su contenido. Asimis- mo, necesitan políticas salariales y sobre ingresos que sean adecuadas, y que se complementen con políticas de fomento del ahorro, la inversión y el desarrollo de la capacidad productiva.

Sin embargo, y en segundo lugar, la excesiva dependencia de la eficacia "automática" de las señales de los precios y de la reforma en el plano macroeconómico se ha traducido en una tendencia a subestimar la debilidad de las instituciones, las fallas de los mercados (muchos son imperfectos, segmentados o incornpletos) y la importancia de las externalidades. En algunos casos esto ha llevado a confiar demasiado en la capacidad de las políticas macroeconómicas para estimular por sí solas el crecimiento. La experiencia ha demostrado que aun las políticas mejor concebidas pueden resultar ineficaces o no dar los efectos deseados en contextos institucionales adversos. De hecho, estos problemas estructurales dificultan el logro de un crecimiento sostenido exclusivamente sobre la base de las señales de los precios.

En consecuencia, las dos lecciones más importantes que nos ha dejado el proceso de reforma son una combinación de un nuevo acercamiento a la ortodoxia (interés en que los precios sean "correctos") y de abandono de la ortodoxia (los precios "correctos" son necesarios, pero no suficientes). Una vez reconocidas las interacciones que se producen entre las medidas de estabilización y las reformas estructurales, es posible que la consideración integral de situaciones macroeconomicas hipotéticas, de las instituciones y los marcos reguladores, de los mercados de factores y del comportamiento microeconómico adquiera más importancia en el proceso de formulación de políticas en el futuro.

\section{Acotación al margen: la experiencia de desarrollo del Asia oriental y sus efectos en América Latina}

A modo de paréntesis, tal vez convenga recordar que ya en la década de 1980 las economías recientemente industrializadas del Asia oriental (Singapur, la República de Corea, la provincia china de Taiwán y también Hong Kong) despertaron un enorme interés en
América Latina, donde se las consideraba ejemplos exitosos que tenían mucho que ofrecer a la región (Fajnzylber, 1981). En efecto, tanto Ios partidarios de la orientación de la economía hacia las exportaciones como quienes abogaban por una amplia intervención 
estatal podían encontrar modelos dignos de imitarse en esos parses. Eso explica la realización de numerosos estudios en los últimos años con el fin de sacar conclusiones prácticas del éxito de las "economías de alto rendimiento de Asia", de los cuales el más conocido es sin duda "The East Asian Miracle" (Banco Mundial, 1993).

Evidentemente, no hay consenso con respecto a lo que podríamos aprender del éxito logrado por las economías asiáticas; los especialistas difieren sobre todo en lo que respecta a la contribución efectiva de la importancia preponderante otorgada a las exportaciones y al mercado, o bien de las políticas industriales (Fishlow y otros, 1994). Además, es probable que la obsesión que han despertado los "tigres"asiáticos, sobre todo la República de Corea, se haya encauzado mal, por to menos en parte, dadas las importantes diferencias culturales, políticas, institucionales y economicas que existen entre ellos mismos $\mathrm{y}$, más aún, entre ellos y los países latinoamericanos. Además, hay que tener presente que en esos "tigres" la transición de la estrategia de sustitución de importaciones a la orientada a las exportaciones se produjo antes de que la globalización redujera considerablemente las posibilidades de maniobrar en el terreno de la formulación de políticas, dado que hoy en día el mundo es menos tolerante que antaño del empleo de mecanismos graduales o selectivos para la liberalización del comercio.

De todos modos, es mucho lo que se puede aprender del proceso de desarrollo de las economfas asiáticas en las últimas décadas, ${ }^{5}$ sobre todo de los países de Asia sudoriental (Indonesia, Malasia, Filipinas y
Tailandia), que tienen muchos elementos en común con varios países latinoamericanos; y obviamente, existen posibilidades de intensificar la cooperación interregional y el intercambio de experiencias. De hecho, en América Latina se están aplicando sistemáticamente algunas de las medidas que, a juicio de la mayoría, explicarían el éxito logrado por las economías asiáticas de alto rendimiento. Entre otras, cabría mencionar la búsqueda de la estabilidad macroeconómica (lo que el Banco Mundial define como "una buena base de apoyo") y el reconocimiento de la necesidad de invertir más en capital humano (que aún no tiene el alcance efectivo de los esfuerzos realizados en ese sentido en Asia oriental), así como la necesidad de aumentar el ahorro financiero interno y de orientar aún más la economfa hacia las exportaciones. Por último, lo observado en numerosos países de Asia oriental también sirve de fuente de inspiración a los partidarios de otorgar a las políticas industriales un papel más amplio en el fomento del desarrollo en América Latina.

Al igual que en nuestra región, en las economías de Asia oriental vemos que también se da una combinación de ortodoxia y heterodoxia. Según el Banco Mundial, "la aplicación de políticas de desarrollo bien concebidas fue uno de los principales factores que hicieron posible el crecimiento ... pero no lo son todo. En la mayor parte de esas economías, el gobierno intervino, de una manera u otra, sistemáticamente y de muchas maneras, para fomentar el desarrollo en general $y$, en algunos casos, el surgimiento de determinadas ramas de actividad" (Banco Mundial, 1993, p. 5).

\section{VI}

\section{Implicaciones futuras de las tendencias actuales}

De mis comentarios anteriores se puede deducir que, a mi juicio, el ciclo ortodoxo del pensamiento económico ya habría llegado a su punto culminante y que poco a poco éste comenzaría a encauzarse nuevamen-

\footnotetext{
${ }^{5}$ No puede considerarse que esos casos conformen un modelo. En su prólogo al estudio del Banco Mundial (1993), Lewis Preston, Presidente del Banco en la fecha de su publicación, sefiala acertadamente que las "ocho economías estudiadns aplicaron combinaciones muy distintas de politicas, que comprendfan desde un liberalismo extrento a un intervencionismo extremo".
}

te en la dirección opuesta. Aún no se sabe si estamos a punto de que se produzca un giro brusco comparable a los dos cambios descritos anteriormente o, lo que parece más probable, ante un ajuste más moderado de las tendencias actuales. Sin embargo, es indudable que el conjunto de ajustes previsibles tendrá profundas repercusiones en lo que respecta a la modalidad de crecimiento y que, a consecuencia de ellos, el desarrollo de la región en la segunda mitad de los años noventa diferirá notablemente del que hemos conocido en los primeros cinco años de la década. 
¿Qué características tendrán esos ajustes y cómo influirán en las tendencias futuras en materia de pensamiento económico y en las políticas relacionadas con el desarrollo? A continuación, plantearé algunas posibilidades, basándome en las ideas que ya he expuesto y evitando incursionar en los temas que probablemente examinará Albert Fishlow en su exposición sobre las perspectivas de desarrollo en América Latina y sus posibles consecuencias.

Para comenzar, quisiera referirme a la idea de que el pensamiento económico está sujeto a ciclos. Es poco probable que la marcada tendencia a la adopción de reformas basadas en el mercado sea sustituida por un paradigma rígido. Por el contrario, algunas de las insuficiencias que presenta el desempeño económico y que con o sin razón se atribuyen únicamente a la aplicación de las políticas de mercado, ya han dado origen a cierto revisionismo, o por lo menos al cuestionamiento de las ideas que gozan de mayor aceptación; sobre todo, nos han llevado a preguntarnos si no será exagerado suponer que los precios "correctos", por sí solos, se traducirán en un crecimiento sostenible con estabilidad financiera y con un mejoramiento sostenido de la equidad.

Lo anterior no significa que esté proponiendo un regreso a políticas caducas del pasado, que serían incompatibles con el actual funcionamiento de la economía internacional. Lo que quiero decir es que cabe suponer que la globalización dictará ciertas normas de comportamiento internacional en cuanto a la formulación de políticas macroeconómicas, lo que probablemente sea preferible, en vista del valor que se le reconoce a la continuidad (por contraposición a las oscilaciones exageradas) como parte de la estabilidad que los países necesitan ofrecer para competir por los recursos y los mercados a nivel mundial. Sin embargo, lo más probable es que, ante todo, se deje de otorgar primordial importancia a la gestión macroeconómica de corto plazo para centrar la atención en las estrategias de desarrollo de mediano plazo; en segundo lugar, es posible que se redefina la contribución de las políticas públicas al logro de los objetivos de desarrollo esenciales, entre otros el incremento de la productividad sistémica y a nivel de las empresas, la sustentabilidad ambiental y la mejor distribución del ingreso. En resumen, es posible que se produzca esa combinación de ortodoxia y heterodoxia a la que me referí antes, es decir, de una gestión macroeconómica estable y coherente, por una parte, y de una nueva modalidad de intervención a nivel microeconómico y mesoeconómico, por atra. Este enfoque, que algunos autores definen como "neoestructuralismo" (Fishlow, 1985; Sunkel, 1991) concuerda en términos generales con los planteamientos que viene presentando la CEPAL desde 1990 en sus principales publicaciones.

Por lo tanto, la orientación y el contenido general de la formulación de políticas cambiarán una vez que se enmarquen en un contexto de mediano plazo más definido. Como he señalado, no se dejará de lado el objetivo de mantenimiento de los equilibrios macroeconómicos, pero probablemente se haga un mayor esfuerzo por evitar que la búsqueda de estabilidad impida una asignación eficiente de los recursos necesarios para elevar el nivel de productividad. La estabilidad macroeconómica no se limita a la baja inflación y al equilibrio fiscal; también supone un déficit sostenible en cuenta corriente, un nivel de ahorro interno que permita realizar inversiones, un tipo de cambio real adecuado y un nivel de demanda agregada congruente con la plena utilización de la capacidad de producción existente. Es de suponer que los responsables de la formulación de políticas se esforzarán por lograr avances simultáneos en todos los frentes, en lugar de centrarse en uno u otro objetivo a costa de los demás.

Lo más probable es que la ortodoxia relativa que caracterizará la gestión macroeconómica sea complementada con un mayor "activismo" de los gobiernos. Este activismo se reflejará en intervenciones más selectivas en materia de políticas macroeconómicas, con miras a lograr determinados objetivos de desarrollo, entre otros adquirir competitividad en el plano internacional, mantener la estabilidad de los precios y alcanzar una mayor equidad social. Por lo tanto, las políticas públicas no se limitarán al mantenimiento de una coherencia macroeconómica global, sino que se hará más hincapié en las políticas de desarrollo productivo. Por ejemplo, es posible que se adopten políticas de apoyo a las redes de información, de fortalecimiento de los mecanismos de cooperación empresarial, de protección de la propiedad intelectual, de estímulo al establecimiento de centros de investigación y servicios de extensión que difundan las tecnologías propias de un determinado sector, de adopción de normas y estándares internacionales, y de promoción de la capacitación y el perfeccionamiento profesional.

También cabe prever que los gobiernos intervendrán en mayor medida en la movilización del ahorro nacional y su orientación a inversiones productivas. El depender exclusivamente del mercado y de las tasas de interés no se ha traducido en una relación adecuada entre el ahorro y el producto interno bruto; además, ha 
impedido lograr una mejor relación entre inversión y PIB. Para que el ahorro y la inversión alcancen el nivel necesario, habrá que complementar la gestión macroeconómica estable con incentivos eficaces e instituciones adecuadas. Es probable que también se haga un mayor esfuerzo por fomentar el ahorro institucional (lo que implicaría la reforma del sistema de pensiones y la concesion de incentivos tributarios), perfeccionar $y$ fortalecer los sistemas financieros, crear instituciones e instrumentos financieros bien regulados y supervisados y facilitar el acceso al capital de inversión a ramas de actividad que antes no lo tenfan.

En segundo lugar, el viraje que se ba producido en la última década, y que ha llevado a proponer una mayor especialización en la exportación de productos básicos industriales e incluso productos primarios, es un arma de doble filo. Por una parte, es razonable que el crecimiento de la región se base en la explotación de su abundante dotación de recursos naturales, especialmente en vista de que la aplicación de innovaciones tecnológicas ha elevado en general la competitividad internacional de la mayoría de dichos recursos $y$, en consecuencia, la región tiene un mayor acceso a los respectivos mercados. Por otra, esta especialización tiene cierta similitud con la división internacional del trabajo que la CEPAL advirtí́ ya en 1949; sería irónico que volviéramos al punto de partida y nos diéramos cuenta de que, en el contexto de la economía mundial, los países en desarrollo (o al menos los de América $\mathrm{La}$ tina y el Caribe) deben especializarse en productos básicos cuya demanda se caracteriza por una baja elasticidad en los mercados mundiales, lo que los limitaría a los sectores de baja productividad. Uno de los principales objetivos de lo que llamamos "transformación productiva" es el desplazamiento hacia actividades económicas que tengan un mayor valor agregado y un potencial de crecimiento más alto, es decir ventajas comparativas dinámicas. Por lo tanto, el objetivo de las intervenciones selectivas a las que me he referido probablemente sea el desarrollo de conjuntos de actividades productivas en torno a los productos básicos industriales que constituyen uno de los puntos fuertes de América Latina en la actualidad, ${ }^{6}$ con eslabonamientos hacia adelante y hacia atrás que permitan ofrecer artículos con un mayor grado de elaboración y valor agregado.

En relación con lo anterior, retomaré una idea que sólo esbocé brevemente; me refiero al lugar cada vez

\footnotetext{
${ }^{6}$ Productos derivados de la pesca, la silvicultura, ta minería, ef petróleo, los necursos energéticos y la agricultura.
}

más destacado que ocuparán las cuestiones ambientales en la agenda de políticas de América Latina en los próximos años. Ello se debe a que muy probablemente el crecimiento dependerá en gran medida de la explotación eficiente y ambientalmente racional de los recursos naturales y su procesamiento (CEPAL, 1991). Además, ya es indudable que el vínculo entre las normas ambientales y el comercio se convertírá en un aspecto cada vez más importante de las relaciones económicas internacionales, y es preferible que los países de la región adopten una estrategia ofensiva en lugar de exponerse a una nueva forma de proteccionismo. En parte gracias al impacto de la Conferencia de las Naciones Unidas sobre el Medio Ambiente y el Desarrollo celebrada en 1992, el antiguo dilema entre crecimiento y proteccion del medio ambiente ha quedado prácticamente superado; hoy se reconoce cada vez más que no son necesariamente incompatibles y que podrían incluso reforzarse entre sí.

En cuarto lugar, otro aspecto de la formulación de políticas cuya importancia se irá acentuando en los próximos ańos serán los intentos por conciliar las medidas unilaterales de liberalización del comercio con acuerdos comerciales formales a nivel intrarregional $\mathrm{e}$ incluso intrahemisférico. Resulta interesante observar que el impulso dado a la cooperación regional en los últimos años no ha menoscabado el compromiso de la mayoría de los países de participar en el proceso de liberalización del comercio a nivel mundial, lo que ha dado resultados relativamente satisfactorios. Sin embargo, así como el regionalismo puede contribuir a crear una economía internacional más abierta, también puede influir en la fragmentación de la economía mundial o regional en bloques. Hipotéticamente, la nueva generación de acuerdos comerciales puede dar origen a una amplia gama de situaciones, desde una región fragmentada en cuatro o cinco agrupaciones subregionales hasta el establecimiento de una zona de libre comercio de alcance hemisférico. No se puede saber aún qué ocurrirá, aunque es de esperar que se concrete esta última posibilidad. En todo caso, no cabe duda de que el tema ocupará un lugar importante en la agenda de desarrollo de la región en lo que resta de esta década y probablemente durante muchos años más.

En quinto lugar, es innegable que la tarea pendiente más importante del período de la posguerra en América Latina es la relativa a la equidad. Dada la creciente disparidad entre los distintos segmentos de la poblacion y los efectos que ésta tiene en lo que respecta a la interacción política en sociedades más pluralistas, no cabe duda de que el problema tendrá que abordarse de 
manera más sistemática. La generación de empleos se convertirá en uno de los objetivos más importantes de las políticas públicas, lo que impone no sólo la necesidad de lograr una mejor relación entre inversión y producto interno bruto y tasas más altas de crecimiento, sino también la de dar incentivos a las actividades que supongan un uso intensivo de mano de obra y a la vez puedan ser competitivas, $y$, en general, la de fomentar una mayor productividad. También es muy posible que se asigne un mayor volumen de recursos a los servicios de salud y, sobre todo, de educación. Se retomará el tema del desarrollo participativo, que ha despertado más interés en Asia que en América Latina (Ghai, 1988; Schneider, 1995). Además, se otorgará cada vez más importancia al perfeccionamiento de la aplicación de las políticas sociales, incluida la focalización de los gastos hacia los segmentos más vuinerables de la población. Incluso es posible que, al menos en algunos casos, resurja el antiguo debate sobre la posible justificación de las reformas agrarias, que prácticamente había desaparecido de la agenda del desarrollo hasta hace poco tiempo, aunque en Brasil vuelve a estar en primer plano.

La inequidad siempre ha sido, indudablemente, un elemento característico del desarrollo de América Latina. Sin embargo, hay razones para creer que es posible lograr auténticos avances en este plano, especialmente si se logran tasas de crecimiento más altas. Pero también hay motivos para actuar con un optimismo cauteloso. En primer lugar, las tasas de crecimiento de la población disminuyen sensiblemente y los efectos que tendrá este fenómeno en la demanda de servicios públicos y en el número de nuevos integrantes de la fuerza laboral se harán sentir en los años venideros. En segundo término, la creciente atención que muchos países han venido prestando en los últimos años a los aspectos cuantitativos y cualitativos de la educación, y las cuantiosas inversiones en capital humano destinadas a evitar que los trabajadores queden a la zaga del progreso técnico, deben comenzar a rendir frutos tangibles a comienzos del nuevo siglo. En tercer lugar, el extraordinario auge de las organizaciones de base en los últimos años, unido a la consolidación de los regímenes políticos democráticos y participativos, deberá diversificar la estructura de los grupos de presión, en los que actualmente predominan las grandes empresas.

Por último, aunque en nuestra región hay escasos partidarios de volver a la intervención gubernamental autoritaria o de la reaparicion de "sectores productivos privilegiados", es evidente que la agenda de políticas públicas será amplia, compleja y exigente. Para que la tendencia a la democratización reciba toda la atención que merece, la reforma del sector público deberá ocupar un lugar destacado en la agenda de desarrollo, con el único objetivo de dotar a los gobiernos de una mayor capacidad para desempeñar su labor con más eficacia, y supuestamente con mayor transparencia y responsabilidad que en el pasado. Esto pondrá fin al debate sobre este tema y la ortodoxia seguirá teniendo defensores, desconfiados por principio, de toda intervención pública. Si queremos ser consecuentes con la idea de que el pensamiento económico atraviesa por ciclos, tendríamos que reconocer que éste puede ser el origen del nuevo giro en materia de políticas, que podría conducirnos, tal vez alrededor del año 2020, a una mayor ortodoxia.

Para terminar con un toque de optimismo, podría decir que si estas tendencias del pensamiento sobre el desarrollo y la formulación de políticas se mantienen, el desempeño de la mayoría de los países de América Latina mejorará en los años venideros, tanto en términos de crecimiento como de equidad. Esto significaría que el proceso de aprendizaje de los años ochenta y noventa puede hacer una valiosa contribución al desarrollo a comienzos del próximo milenio. El pragmatismo que caracteriza hoy en día a la formulación de políticas debería ser un importante componente de ese proceso. Lo mismo se podría afirmar con respecto a la creatividad y al espíritu empresarial cada vez más acentuados que ha exhibido el sector privado de la región en los últimos años. Asimismo, la intensificación de la interdependencia económica dentro de la región, estimulada por una nueva generación de acuerdos formales de integración, mitigará su vulnerabilidad a las crisis externas. En el año 2000 América Latina y el Caribe tendrán una población de $515 \mathrm{mi}$ llones de habitantes con niveles medianos de ingreso per cápita (alrededor de 3800 dolares en promedio), lo que convertirá a la región en un atractivo mercado.

Sin duda, el progreso no será lineal, ni tampoco beneficiará a todos por igual, ya se trate de países, regiones o estratos de la población. Además, pasará mucho tiempo antes de que se superen muchos de los problemas que afectan a la región y que hoy parecen insolubles: la expansión urbana descontrolada, la inseguridad individual, la destrucción del medio ambiente, la pobreza generalizada y el deterioro de la infraestructura. Sin embargo, no se puede negar que en general nuestros pasos están bien encaminados. 


\section{Bibliografía}

Banco Mundial (1993): The East Asian Miracle: Economic Growth and Public Policy, Oxford, Oxford University Press.

Birdsall, N. y C. E. Lozada (1996): Recurring themes in Latin American economic thought: From Prebisch to the market and back, Securing Stability and Growth in Latin America: Policy Issues and Prospects for Shock-prone Economies. R. Hausmann y H. Reisen (comps.), París, Organización de Cooperación y Desarrollo Económicos (OCDE).

Bruno, M. y atros (comps.) (1988): Inftation Stabilization: The Experience of Israel, Argentina, Brazil, Bolivia and Mexico, Cambridge, Massachusetts, The MIT Press.

CEFAl (Comisión Económica para América Latina y el Caribe) (1996в): Fortalecer el desarrollo. Interacciones entre macro y microeconomia, LC/G.1898(SES.26/3). Santiago de Chile.

(1996b): Quince años de desempeño económico. América Latina y el Caribe, 1980.1995, LC/G.1925/Rev. 1-P, Santiago de Chile. Publicación de las Naciones Unidas, $\mathrm{N}^{a}$ de venta S.96.II.G.3.

(1995a): Panorama social de América Latina. Edición 1995, LC/G.1886-P, Santiago de Chile. Publicación de las Naciones Unjdas, $\mathrm{N}^{0}$ de venta S.95.II.G.17.

(1995b): América Latina y el Caribe: politicas para mejorar la inserción en la economia mundial. LCG.1800/Rev.I-P, Santiago de Chile. Publicación de las Narciones Unidas, $\mathrm{N}^{\circ}$ de venta S.95.1I.G.6.

(1994): El regionalismo abierto en América Latina y el Caribe. La integración econbmica al servicio de la transformación productiva con equidad. LC/G.1801/Rev.1-P, Santiago de Chile. Publicación de las Naciones Unidas, $N^{\circ}$ de venta S.94.II.G.3.

(1992): Equidad y transformacion productiva: un enfoque integrodo, LC/G.1701/Rev.1-P, Santiago de Chile, abril Publicación de las Naciones Unidas, $\mathrm{N}^{\circ}$ de venta S.92.II.G.S.

(1991): El desarrollo sustentable: transformacion productiva, equidad y medio ambiente, LC/G.1648/Rev .2-P, Santiago de Cbile, mayo, Publicación de las Naciones Unidas, $\mathrm{N}^{\circ}$ de venta S.91.II.G.5.

(1990): Transformación productiva con equidad. La tarea prioritaria del desarrollo de América Latina y el Caribe en los años noventa, LC/G.1601-P, Santiago de Chile, marzo. Publicación de las Naciones Unidas, $N^{0}$ de venta, S.90.II.G.6.

(1951): Estudio económico de América latina 1949 ECN/12/164/Rev.1, Nueva York. Publicación de las Naciones Unidas, $\mathrm{N}^{\mathrm{a}}$ de venta, 1951.II.G.I.

Corbo, V. (1988): Problemas, teoría del desarnollo y estrategias en America Latina, Estudios púbicos, $\mathrm{N}^{\circ} 32$, Santiago de Chile.

Corden, M. W. (1966): The structure of a tariff system and the effective protective rate, Journal of Political Economy, vol, $74, N^{0} 3$.

Dorubusch, R. y S. Edwards (1989): The Macroeconomics of Populism in Latin America. Policy, Planning, and Research Working Papers, $\mathbf{N}^{\mathrm{o}}$ 316, Washington, D.C., Banco Mundial.

Dornbusch, R. y S. Fischer (1993): Moderate inflation, The World Bank Economic Review, vol. 7, N 1, Washington, D.C., enero.

Dornbusch, R. y A. Werner (1994); Mexico: Stabilization, reform and no growth, Brookings Papers on Economic Activity, $\mathrm{N}^{\circ}$ 1, Washington, D.C.
Edwards, S. (1996): The Disturbing Uaderperformance of the Latin American Economies, Interamerican Dialogue Plenary Meeting, Washington, D.C., mayo.

Fajnzylber, F. (1981): Reflexiones sobre la industrialización exportadora del sudeste asiático, Revista de la CEPAL, $N^{*} 15$ (E/CEPAL/G.1 187), Santiago de Chile, diciembre.

Fishlow, A. (1985): The state of Latin American economics, Economic and Social Progress in Latin America. Report. Washington, D.C., Banco Interamericano de Desarrollo (BID).

Fishlow, A. y otros (1994): Miracle or Design? Lessons from the East Asian Experience, Policy Essay N $N^{\circ} 11$, Washington, D.C., Consejo de Desarrollo de Ultramar.

Ffrench-Davis, R., D. Titelman y A. Uthoff (1994): International competitiventess and the macroeconomics of capital account opening Serie documentos de trabajo, $\mathrm{N}^{\circ} 29$, Santiago de Chile. Comisión Econónica para América Latina y el Caribe (CEPAL).

Ghai, D, (1988): Participatory development: Some perspectives from grass-roots experiences, discussion paper, $N^{0} 5$, Ginebra, Instituto de Investigaciones de las Naciones Unidas para el Desarrollo Social (UNRISD).

Krugman, P. (1992): Towards a counter-counterrevolution in development theory, Proceedings of the World Bank Annual Conference on Development Economics, Washington, D.C.. Banco Mundial.

Morales, J. A. y J. Sachs (1988): Bolivin's econonic crisis, en J.D. Sachs (comp.), Developing Country Debt and the World Economy, Chicago, University of Chicago Press.

Ramos, J. (1986): Neoconservative Economics in the Southern Cone of Latin America, 1973-1983, Baltimore, The Johns Hopkins University Press.

Sachs, J., A. Tornell y A. Velasco (1995): The collapse of the Mexican peso: What have we learned, Discussion Paper $\mathbf{N}^{\mathbf{}}$ 1724, Cambridge, Massachusetts, Harvard Institute of Econonic Research.

Schneider. H. (1995): Participatory Development: From Advocacy to Action, París, Centro de Desarrollo de la Organización de Cooperación y Desarrollo Económicos (OCDE).

Scobie, G. (comp.) (1990): Macroeconomic Policy Reformts, Poverty and Nutrition, Ithaca, Cornell University.

Sunkel, O. (comp.) (1991): El desarrollo desde dentro: un enfoque neoesructuralista para la América Latina, México, D.F., Fondo de Cultura Económica (FCE).

Taylor, L. (1992): Polonius lectures again: The World Development Report, the Washington Consensus, and how neoliberal sermons won't solve the economic problems of the developing world, The Bangladesh Development Studies, vol. 20, № 2 y 3.

(1988): Varieties of Stabilization Experience: Towards Sensible Macroeconomics in the Third World Oxford, Clarendon Press.

Viner, J. (1953): International Trade and Economic Development, Oxford, Clarendon Press.

Williamison, J. (1991): Policy reform in Latin America in the 1980s, Structural Adjustment; Retrospect and Prospect, Washington, D.C., American University.

(1990): What Washington means by policy reform, Latin American Adjustment: How Much Has Happened?. John Williamson (comp.). Washington, D.C., Institute for Intemational Economics. 